\title{
LA ESCRITURA COMO PROCESO: METODOLOGÍA PARA LA ENSEÑANZA DE LA EXPRESIÓN ESCRITA EN ESPAÑOL COMO SEGUNDA LENGUA
}

\author{
Marta Madrigal Abarca
}

\begin{abstract}
RESUMEN
En este trabajo se presentan los resultados de una investigación en la cual se propuso una estrategia pedagógica de la enseñanza de la escritura del español a estudiantes no nativos de esta lengua, enfocando el proceso y no el producto. Esta metodología se centra en las distintas actividades de pensamiento superior que realiza un autor para componer un escrito en un período que abarca, desde que se crea una circunstancia social que exige producir un texto, hasta que este se da por acabado. En este enfoque no solamente se muestra el producto terminado, la versión final, sino que lo más importante es describir y aprender los pasos intermedios y las estrategias que deben utilizarse durante el proceso de redacción. De esta manera, el proceso incluye tres etapas: la preescritura, el borrador y la revisión, y se da vital importancia al papel que cumplen tanto los aprendices como los profesores.
\end{abstract}

Palabras clave: escritura, proceso, preescritura, borrador, revisión.

\begin{abstract}
In this course I will present the results of an investigation into a pedagogical strategy for the teaching of written Spanish to non native students. The outcome of which is focused on the process rather than the product. This methodology is centered on distinct upper level thinking activities used by the writer to compose a text. It takes into account the complete period time, which begins when a circumstance is created which stimulates the idea for the production of the text and all the way through until it is finished. Within the scope of this process the final version of the text or product is demonstrated, but more important is the description and the learning of all the intermediate steps and strategies which can be utilized in the writing. In this way the process includes three stages: the pre-writing phase, first drafts and revisions. There is equal and vital importance given to the roles played by both students and teachers in this process.

Key words: writing, process, prewriting, draft, revision.
\end{abstract}

M.L. Marta Madrigal Abarca. Magíster en Español como Segunda Lengua. Profesora de la Escuela de Filología, Lingüística y Literatura de la Universidad de Costa Rica, San Pedro, San José, Costa Rica.

Correo electrónico: madrigal_ma@yahoo.com.mx

Recepción: 26- 2- 2009

Aceptación: 24- 3- 2009 


\section{Introducción}

La habilidad de aprender a escribir es una de las tareas más difíciles que un estudiante debe enfrentar, al tener que vencer obstáculos como la ansiedad y la frustración que muchas veces conlleva el desarrollo de esta destreza. El hecho de comunicar claramente las ideas en forma escrita puede ser un proceso lento y, en muchos casos, agotador. Esta problemática se ve recrudecida para los que quieren aprender a escribir en una lengua extranjera, porque no solamente deben saber manejar la estructura y la forma de la lengua que no es la propia, sino que también tienen que tener conocimiento de un conjunto de reglas de adecuación, coherencia y cohesión. De manera que el desarrollo de la expresión escrita requiere esfuerzos conscientes y deliberados para escoger tanto la forma como el contenido.

Como el proceso de llevar a la forma escrita conceptos, pensamientos e ideas se vuelve muy complejo, ya que un texto escrito representa el producto de una serie de operaciones mentales complicadas, es de vital importancia brindar a los estudiantes las técnicas que les ayuden a conocerse para que puedan desarrollar sus propias estrategias de escritura con el propósito de que el aprendizaje sea exitoso.

\section{Justificación y objetivos}

La lengua escrita no es una habilidad que se puede adquirir de forma espontánea, sino que se vale de otras estrategias que el educador puede brindar a los alumnos con el propósito de que descubran sus propias formas de escritura. Esta situación es una justificante para que profesores, autores o investigadores en segundas lenguas hayan hecho publicaciones de libros y manuales que enseñan a sus estudiantes esta destreza. Sin embargo, este material no está basado en una estrategia metodológica acorde con las necesidades de aprendizaje de cada estudiante. Así, los recursos bibliográficos utilizados para la enseñanza de la escritura carecen de planteamientos teórico-metodológicos que desemboquen, de manera lógica y ordenada, en las situaciones de aprendizaje o actividades para los distintos contenidos.

El presente proyecto consistió en diseñar una metodología para enseñar a escribir diferentes tipos de textos en español a estudiantes no nativos de esta lengua. El objetivo primordial fue que los estudiantes aprendieran algunas estrategias para la redacción de ciertos tipos de textos de estructura descriptiva, narrativa, expositiva y argumentativa con énfasis en el proceso.

\section{Marco teórico}

\subsection{El enfoque al proceso}

Los investigadores de la expresión escrita están de acuerdo en que, si bien el producto final es importante, también lo es el proceso por medio del cual se llega a ese último resultado. Sin embargo, en muchos casos, este proceso no es tomado en cuenta. Brookes y Grundy expresan que:

A todos nosotros (y esto incluye a nuestros estudiantes) nos gustaría elaborar productos finales que sean imaginativos, seguros, personales y públicos fluidos y sin errores. Algunos maestros les piden a sus estudiantes crear una serie de productos y esperan que la repetición constante ayudada por el cuidadoso señalamiento y evaluación resultarán eventualmente en un aceptable producto no siempre dándose cuenta de cuan interesante y efectivo puede ser el proceso (1998: 15). 
Debido a esta situación, muchos de los estudiosos y profesores prefieren abordar la escritura con un modelo que enfatice el aspecto cognitivo que entra en juego en el acto de la escritura. Este modelo, denominado Enfoque al Proceso, se centra en las distintas actividades de pensamiento superior, que realiza un autor para componer un escrito, en un período que abarca, desde que se crea una circunstancia social que exige producir un texto, hasta que este se da por acabado.

De esta manera, el texto escrito no es visto como un producto acabado, sino como un proceso, el cual involucra tanto los elementos que proceden del exterior, por ejemplo, las circunstancias que motivan o inducen a escribir, como los elementos específicos que se dan en el interior de la mente del escritor.

El modelo más divulgado es el de Flower y Hayes (1980 y 1981), el cual concibe la composición como una acción dirigida a la consecución de objetivos retóricos. Identifica también tres procesos básicos: la planificación, la redacción y la revisión, que a su vez dan origen a otros subprocesos como la generación de ideas, la formulación de objetivos y la evaluación de producciones intermedias, entre otros.

Según Brookes y Grundy (1998), el modelo del Enfoque al Proceso brinda importantísimos aportes a la enseñanza de la escritura, ya que es de gran utilidad para los profesores el poder analizar los diferentes elementos implicados en los fragmentos escritos por sus estudiantes, pues esto ayudaría a que los aprendices se volvieran conscientes de estos elementos y usarían este conocimiento positivamente a la hora de realizar sus composiciones. Para estos autores, el proceso de escritura se da: "Uniendo uno por uno los elementos que determinan lo que escribimos" (1998: 7).

\subsubsection{Las fases en el enfoque al proceso}

El énfasis en el proceso se enfoca en los pasos intermedios y en las estrategias que deben utilizarse durante todo el proceso de la redacción de un escrito; de esta manera, se desarrollan y evalúan tres etapas fundamentales: la preescritura, el borrador y la revisión. La primera fase, la preescritura, involucra la búsqueda de temas; se trata de encontrar ideas sobre lo que se va a escribir, cómo van a interactuar y cómo se desarrollan y se organizan. La segunda fase es el borrador. En este estadio se obtienen las ideas ya formadas y se escriben de manera preliminar; es decir, en borrador. La última fase es la de revisión, la cual involucra diferentes actividades para evaluar lo que ha sido escrito; sin embargo, es importante tomar en cuenta que esta fase puede ocurrir en cualquier tiempo durante el proceso de composición y no solo como etapa final.

\subsubsection{El papel de los aprendices y de los profesores}

En este enfoque, el rol de los aprendices y el de los profesores es muy particular, ya que el énfasis se pone en el alumno y no en el texto escrito. Es decir, se enseña al estudiante a pensar, a hacer esquemas, a ordenar las ideas, a pulir la estructura de las oraciones, a revisar el escrito.

El papel del profesor es igualmente redefinido y negociado. Más que intentar obligar a los aprendices a asegurarse de producir una escritura correcta, los maestros actúan como facilitadores, organizando experiencias de escritura que le permitan al aprendiz desarrollar estrategias de composición efectivas. El profesor orienta y asesora el trabajo del alumno por medio de la observación y de la discusión para identificar las técnicas adecuadas para cada uno, de manera que pueda integrarlas y adaptarlas a su forma de trabajar. Para el profesor es muy importante el análisis individual de las necesidades del cada estudiante, por lo tanto, no 
propone modelos a seguir, sino que es fundamentalmente un guía, el cual facilita los elementos básicos para que los estudiantes encuentren sus propias estrategias que le ayuden en el proceso de escritura. Al respecto, Silva, citado por Brookes y Grundy (1998: 8), dice:

\footnotetext{
El rol del profesor es ayudar al estudiante a desarrollar estrategias viables para poder empezar (encontrando temas, generando ideas e información, enfocando y planeando estructuras y procedimientos), para esbozar (motivando múltiples borradores), para revisar (agregando, eliminado, modificando, arreglando ideas) y para editar (atendiendo el vocabulario, la estructura de la frase gramatical y la mecánica).
}

Parafraseando a Richards (1990: 114), un exitoso profesor de escritura no es aquel que ha desarrollado un "método" de enseñanza, sino aquel que crea un ambiente efectivo para el aprendizaje, en donde los escritores principiantes se sientan cómodos y puedan explorar la naturaleza de la escritura, al descubrir sus propias fortalezas y debilidades como escritores. Y es a través de todo el proceso que los roles, tanto de los profesores como de los aprendices y de los patrones de interacción entre ellos, deberán ser negociados constantemente.

\subsubsection{Tipos de actividades de enseñanza en el enfoque al proceso}

Las actividades relacionadas con la fase de preescritura son diseñadas para ayudar a los aprendices a generar planes y desarrollar ideas para la redacción. Entre estas actividades, los investigadores incluyen: los diarios, la lluvia de ideas, la asociación libre, mapas de palabras, escritura rápida y diversas actividades para reunir información como artículos de periódicos, películas, canciones, entre otras.

Las actividades relacionadas con la fase del borrador se dedican al intento inicial de la elaboración del bosquejo de las diferentes secciones de la composición para completar el borrador del escrito. El esquema puede ser desarrollado a partir de preguntas estratégicas, por ejemplo: ¿qué quiere escribir realmente?, ¿cuál es la meta?, ¿cuál es la actitud hacia esa tarea?, ¿qué sabe sobre ese tema?, ¿qué necesita todavía averiguar del tema?, ¿cuáles ideas parecen quedar juntas?, ¿qué es lo más importante del tema?, ¿quién quisiera leer lo que se va a escribir?, etc. También en esta fase se pueden incluir actividades como: la escritura con énfasis en el tiempo, el párrafo desordenado o el escrito desordenado, la redacción de tesis y tema, la escritura rápida y el borrador en grupo.

Por último, la fase de la revisión involucra actividades que corresponden a la edición y la corrección de pruebas. Estas incluyen la retroalimentación entre compañeros, los ejercicios de reescritura, la retroalimentación del profesor y el chequeo por medio de listas.

\section{Marco metodológico}

Se trabajó con una población de 14 estudiantes de Estados Unidos, adultos, estudiantes de universidades estadounidenses de nivel avanzado, según la escala de ACTFUL (American Council on the Teaching of Foreing Languages).

Con el propósito de mostrar los resultados de la investigación, los estudiantes realizaron una composición de diagnóstico antes de iniciar el curso y otra al final del curso. Ambas composiciones fueron evaluadas con la misma escala de valores para determinar el progreso.

Para evaluar cada fase del proceso (preescritura, borrador y revisión) se seleccionaron las actividades más relevantes de cada una de ellas y se midieron con una escala de frecuencia. Dicha escala está organizada de mayor a menor de la manera siguiente: siempre, casi siempre, a veces y nunca. Los datos relativos a cada estado se detallan en gráficos separados, de manera que el gráfico No. 1 corresponde a la fase de la preescritura; el No. 2, a la fase del borrador, 
y el No. 3 muestra los resultados de la fase de revisión. En el eje vertical, a la izquierda de cada gráfico, se indica el número de estudiantes. En el eje horizontal, abajo de cada gráfico, se especifica la frecuencia.

\subsection{Tipos de ejercicios y actividades}

La tarea central de cada clase fue la redacción de textos de estructura descriptiva, narrativa, expositiva y argumentativa. Se practicaron también las destrezas del habla, de lectura y de escucha; estas destrezas fueron desarrolladas mediante diversas actividades, tales como: escucha de párrafos y textos, cuentos y canciones, lecturas de temas específicos, proyección de videos, conversación sobre temas varios, concursos, debates, participaciones grupales para la creación de lluvia de ideas y para la revisión y la retroalimentación entre los estudiantes.

Para apoyar la destreza escrita se realizaron ejercicios controlados como completes, de “cloze", sustitución y selección múltiple. En las prácticas guiadas se trabajó con la redacción de oraciones y párrafos a partir de fotografías, construcción de bosquejos basados en preguntas, creación de diferentes tipos o modelos para la generación de ideas, asociación de imágenes con el texto escrito, completar esquemas narrativos, ordenación de textos a partir de párrafos desordenados, prácticas de pronunciación y dictados.

\subsection{Enfoques metodológicos}

a. El método comunicativo (basado en Los métodos de enseñanza de idiomas de Aquilino Sánchez 1997).

b. El enfoque por tareas (basado en Currículo Development in Language Teaching de Jack C. Richards 2001).

\section{Resultados y análisis}

\subsection{La fase de preescritura}

En esta etapa, el escritor busca el tema del cual va a escribir. Se trata de encontrar ideas sobre el tema, pensar cómo se organizan y se desarrollan y cómo pueden interactuar entre sí. En esta fase se evalúan los siguientes aspectos: búsqueda y desarrollo de ideas, utilización de técnicas diferentes para generar ideas, anotación de las ideas directamente con palabras y expresiones que puedan entender los lectores, la anotación de las ideas, primero para sí mismo y luego con explicación.

Gráfico 1. Evaluación de la fase de preescritura

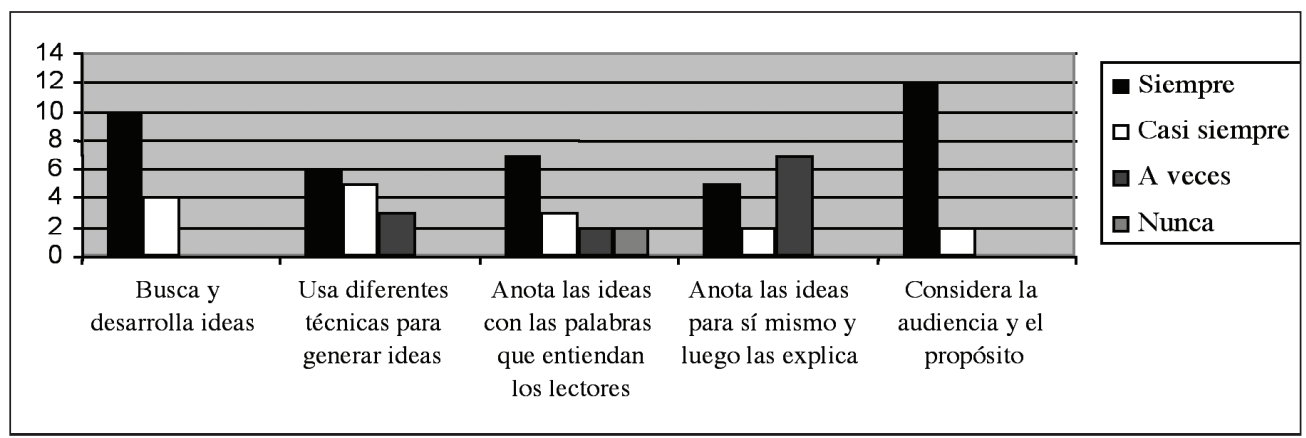




\subsubsection{Busca y desarrolla ideas}

Para algunos estudiantes, la búsqueda de un tema para escribir una composición es a veces un proceso lento y hasta frustrante si no se cuenta con los recursos. Por esta razón, el enfoque al proceso recomienda el uso de diferentes técnicas para desarrollar estrategias que le ayuden al estudiante a encontrar una amplia gama de posibilidades sobre temas acerca de los cuales podría escribir.

En el gráfico 1 se observa que diez de los estudiantes, que representan un $71,42 \%$, siempre utilizaron los recursos que se les brindaron para buscar ideas; mientras que cuatro estudiantes, con un porcentaje de 28,57\% lo hicieron casi siempre. En las frecuencias a veces y nunca no se reportaron estudiantes.

\subsubsection{Usa diferentes técnicas para generar ideas}

La generación de ideas funciona como un mecanismo de estímulo inicial al proceso de escritura, ya que brinda al estudiante la motivación necesaria para empezar a escribir. Durante el curso se implementaron varias actividades para tal propósito, entre las cuales están: gráficos de estrellas, árbol de ideas, rueda de palabras, diagramas y la escritura automática.

Al respecto, del gráfico No. 1 se desprenden las siguientes observaciones: primero, si los estudiantes tenían a su disposición diferentes maneras de generar ideas se esperaría que el 100\% de ellos las hubieran aprovechado; sin embargo, se observa que solamente seis de los estudiantes, con un $42,85 \%$, siempre utilizaron diferentes técnicas para la generación de ideas, mientras que cinco estudiantes, con un $35,1 \%$, lo hicieron casi siempre. En la frecuencia a veces se reportaron tres estudiantes, con un 21,42\%, y en la frecuencia nunca no se reportó ningún estudiante.

En resumen, los datos arrojan que, si bien no todos los estudiantes utilizaron diferentes técnicas para generar ideas, todos utilizaron alguna de ellas. Al revisar el material elaborado por los estudiantes, se podría conjeturar que los estudiantes se sintieron más cómodos con la rueda de palabras y la escritura libre.

\subsubsection{Anota las ideas directamente con palabras y expresiones que puedan entender los lectores}

Al apuntar las ideas que luego serán desarrolladas, el escritor tiene la alternativa de escribir de manera directa sus pensamientos, buscando las palabras que puedan entender los lectores o anotar las ideas tal como se le vienen a la cabeza y después tomar el tiempo para modificarlas de manera que el lector las pueda entender.

En el gráfico No.1 se puede observar que, de los catorce estudiantes, la mitad (50\%) siempre apuntaba las ideas directamente, mientras que tres de ellos, con un 21,42\%, lo hacían casi siempre. En las frecuencias a veces y nunca se registran porcentajes de 14,28\% respectivamente. Es probable que estos estudiantes anotaran primero las ideas para sí mismos y luego las explicaran de manera que el lector las entendiera.

\subsubsection{Anota las ideas para sí mismo y luego busca la mejor manera de explicarlas}

Este aspecto se encuentra en estrecha relación con el anterior. Al observar los datos se nota que, de los catorce estudiantes, el $50 \%$ se ubicó en la frecuencia a veces, y cinco, con un porcentaje de $35,71 \%$, se ubicaron en la frecuencia siempre. Asimismo, solo dos de ellos, con 
un porcentaje de 14,28\%, ocupan la frecuencia de casi siempre. Es decir, si se comparan estos datos con los del apartado anterior, se podría suponer que ese $50 \%$ que a veces anota las ideas para sí mismo siempre prefiere anotar las ideas directamente con palabras que pueda entender el lector. Este comportamiento no es extraño, ya que los escritores tienen las dos posibilidades. La elección de una de las dos estrategias depende no solo de su preferencia, sino también de otras condiciones, como el tiempo del que se dispone, el propósito y la motivación, entre otras.

\subsubsection{Considera el propósito y la audiencia}

Para muchos estudiantes, el propósito es un elemento difícil de determinar, ya que no siempre es obvio. Por esto, en el enfoque al proceso se enfatiza la necesidad de establecer claramente para qué y por qué se quiere escribir. Cada tarea debe tener un propósito y este, debe tener un significado; de este modo, la práctica de escritura será mucho más fácil para el estudiante. De igual manera, al establecer el propósito, es más fácil establecer la audiencia, es decir, quién o quiénes van a leer lo que se va a escribir.

Los datos del gráfico No. 1 muestran que el mayor porcentaje (85,71\%), lo ocupó la frecuencia siempre, mientras que solo dos de los estudiantes, con un 14,28\% lo hizo casi siempre. En otras palabras, la gran mayoría de los estudiantes comprendieron que todo tipo de texto escrito debe tener claramente establecidos tanto el propósito como la audiencia para evitar ambigüedades $y$, de este modo, lograr el efecto que el escritor espera.

\section{La fase de borrador}

Una vez cumplida la fase de la preescritura, el escritor pasa a la siguiente etapa: la realización del borrador. El estudiante tiene que tener claro que escribir no es solamente poner las ideas por escrito en un papel en blanco, sino que los textos escritos que él lee han pasado por uno o varios borradores y que su autor ha tenido que trabajar mucho para conseguir el producto final: ha hecho listas de ideas, ha elaborado esquemas, ha corregido una o varias veces.

En esta fase se consideran los siguientes criterios: elaboración de planes o esquemas, la escritura de más de un borrador, la selección de la información pertinente, la organización de textos en párrafos, la estructuración lógica de los datos, el empleo de las oraciones temáticas y el uso correcto de los signos de puntuación.

Gráfico 2. Evaluación de la fase de borrador

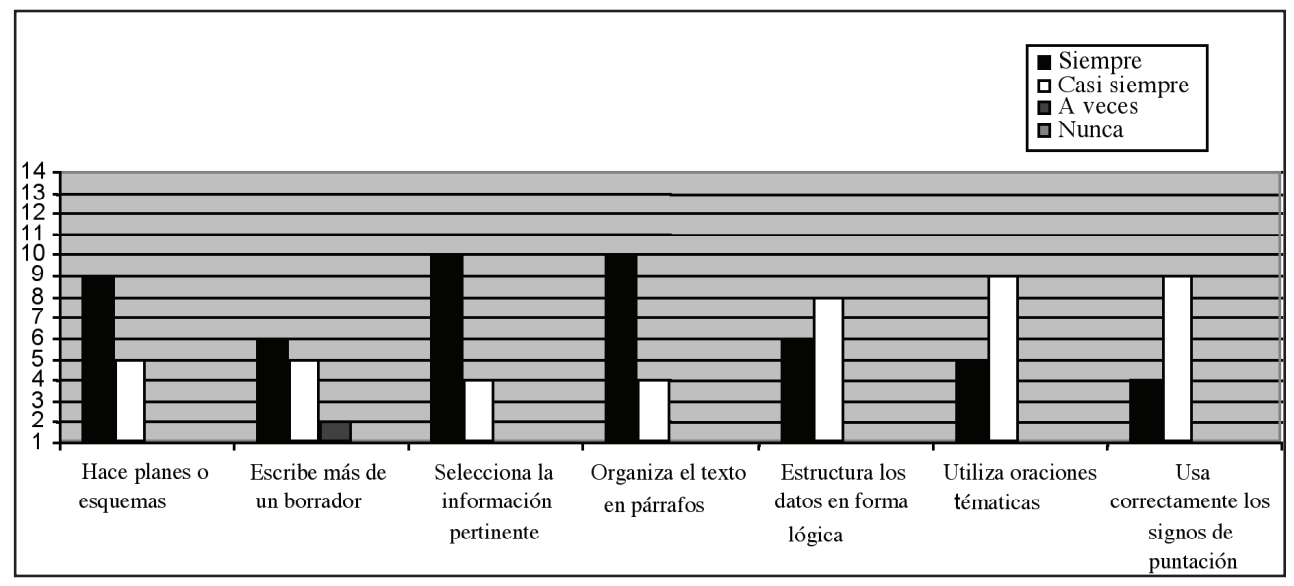




\subsection{Hace planes del texto o esquemas}

En la etapa de preescritura, el estudiante encuentra las ideas que quiere desarrollar en el borrador. No obstante, es en la fase del borrador que se debe planear todo el texto; aquí se trata de organizar las partes del escrito, las ideas importantes, la secuencia de los párrafos. El plan o esquema es como el esqueleto del escrito, el cual se va rellenando en el transcurso de la redacción. Si se cumple con este paso, completar la tarea de escritura es más fácil y rápido.

En el gráfico No. 2 se observa que nueve de los estudiantes, con un porcentaje de $64,28 \%$, siempre hicieron planes o esquemas del texto; mientras que solo cinco de ellos, con un porcentaje de $35,71 \%$, lo hizo a veces. Estos datos evidencian que los estudiantes lograron asimilar que la elaboración de planes previos les ayudaba a escribir mejor sus redacciones.

\subsection{Escribe más de un borrador}

Según el enfoque al proceso, el estudiante debe tener claro que, para ser un escritor competente, no solo se debe planear el escrito, sino que también se debe escribir primero un borrador. Sin embargo, se considera que el buen escritor no se conforma con un solo borrador; por el contrario, escribe tantos borradores como crea necesario.

De acuerdo con los datos arrojados por el gráfico No. 2, seis de los catorce estudiantes, con un porcentaje de 42,85, siempre escribieron más de un borrador, mientras que cinco, correspondiente al 35,71\%, lo hizo casi siempre. Solamente uno de los estudiantes, que representa un 7,14\%, lo hizo a veces. Sin embargo, este porcentaje no es considerado significativo para la evaluación de esta fase.

\subsection{Selecciona la información pertinente}

Se supone que, en el período de generación de ideas, el estudiante tiene a su disposición una gama bastante amplia de ellas. Pero no todas van a ser útiles para trasmitir el mensaje que se desea, sino que deben pasar por un proceso de selección; es decir, se debe pensar en cuáles son más apropiadas de acuerdo al título del texto, a la tesis propuesta, a las oraciones temáticas, etc. Por eso es muy importante que el estudiante aprenda a discernir cuáles son las ideas más convenientes, cuáles debe desechar y cuáles debe elaborar con más detalle para que el escrito logre coherencia y unidad.

Al respecto, el gráfico arroja resultados muy positivos, ya que diez de los catorce estudiantes, con un porcentaje del 71,42, siempre seleccionaron la información apropiada, mientras que la frecuencia casi siempre obtuvo el $28,57 \%$, es decir, solamente cuatro estudiantes no consideraron este criterio a la hora de hacer sus borradores.

\subsection{Organiza el texto en párrafos}

El párrafo puede estar formado por una sola oración gramatical o por varias oraciones temática y semánticamente relacionadas entre sí; es decir, vinculadas por el tema y por el sentido del mensaje. Las oraciones contenidas en el párrafo, además de estar gramaticalmente bien estructuradas, deben cumplir con los requisitos de coherencia y unidad de sentido, indispensables para que el mensaje sea claro y fácilmente comprensible. 
Según los datos del gráfico No. 2, la mayoría de los estudiantes hicieron divisiones del texto. Los resultados muestran los siguientes porcentajes: el $71,42 \%$, que corresponde a diez estudiantes, ocupó la frecuencia siempre, mientras que la minoría, con un porcentaje del 28,57, correspondiente a solo cuatro estudiantes, se ubicó en la frecuencia casi siempre.

\subsection{Estructura los datos en forma lógica}

Al elaborar el plan o esquema, y al seleccionar las ideas pertinentes, es más probable que para el estudiante sea más fácil ordenar la información en forma lógica, ordenada y coherente, sin perder de vista el objetivo que se ha fijado y las características de la audiencia.

En el gráfico No. 2 se evidencia que no todos los estudiantes lograron dominar esta estrategia: de los catorce estudiantes solo seis, que representan un 42,85\%, ocuparon la frecuencia siempre, mientras que ocho, correspondiente a un $57,14 \%$, lo hicieron a veces. Este comportamiento es interesante, ya que, aunque la mayoría de los estudiantes siempre hizo planes y seleccionó las ideas más apropiadas, no supo cómo darles un orden lógico. Esto podría deberse a dos situaciones: o que el estudiante, en la etapa de revisión, no pudo percibir este problema o que el poco tiempo del que disponía interfirió para tomar en cuenta este aspecto de la redacción.

\subsection{Utiliza oraciones temáticas}

Las oraciones temáticas establecen el punto principal del párrafo. Su función es marcar las intenciones del escritor de manera que sean claras para el lector; por esta razón, es importante que el estudiante las utilice, ya que le ayudan a organizar la información del párrafo y, a su vez, la organización lógica de todo el texto.

En el gráfico No.2 se observa que solamente cinco de los estudiantes, con un porcentaje de 35,71, siempre utilizaron las oraciones temáticas, mientras que nueve, es decir, la mayoría, la utilizaron casi siempre. Esta frecuencia marca un porcentaje del 64,28. De esta información surge la interrogante de por qué los estudiantes no utilizaron la oración temática con mayor frecuencia si incluso se hicieron prácticas específicas de ese tema. Esto hace pensar que en dos posibles razones: que la práctica hecha durante el curso fuera insuficiente, o que el tiempo necesario para que el estudiante asimilara este conocimiento no fuera el adecuado.

\subsection{Usa correctamente diversos signos de puntuación}

Para que un texto sea claro y tenga coherencia y sentido de unidad debe estar debidamente puntuado, es por esto que en una clase de composición se insiste en este aspecto. Sin embargo, no todos los estudiantes asimilan este conocimiento.

Esta situación se observa en el gráfico No.2, el cual muestra que, de los catorce estudiantes, un porcentaje del 64,28, casi siempre se utilizó los signos de puntuación apropiadamente, mientras que solamente cuatro, que corresponde a un 28,67\%, lo hicieron siempre. El aspecto de la puntuación también fue evaluado en el producto o redacción final. En esos datos se puede observar que, si bien es cierto hubo una mejoría en el uso de la puntuación, la mayoría de estudiantes no logró obtener calificaciones excelentes. Se podría conjeturar que las prácticas realizadas no fueron suficientes para que los estudiantes lograran adquirir este conocimiento. 


\section{La fase de revisión}

En esta fase se evalúa lo que ha sido escrito. Sin embargo, es importante recalcar que este proceso puede realizarse en cualquier momento durante el proceso de composición y no solo como etapa final. La revisión se puede realizar mediante diversas actividades tales como: retroalimentación del profesor y de los otros estudiantes, autocorrección, corrección en grupo, ejercicios de reescritura, chequeo de listas, entre otras.

Para efectos de esta investigación se evaluaron las siguientes actividades: la revisión tanto de la forma como del contenido del texto, el uso de pautas de autocorrección o de listas de cotejo, la interacción con los compañeros, la aceptación de las correcciones hechas por sus compañeros y la aceptación de las correcciones hechas por el profesor.

Gráfico 3. Evaluación de la fase de revisión

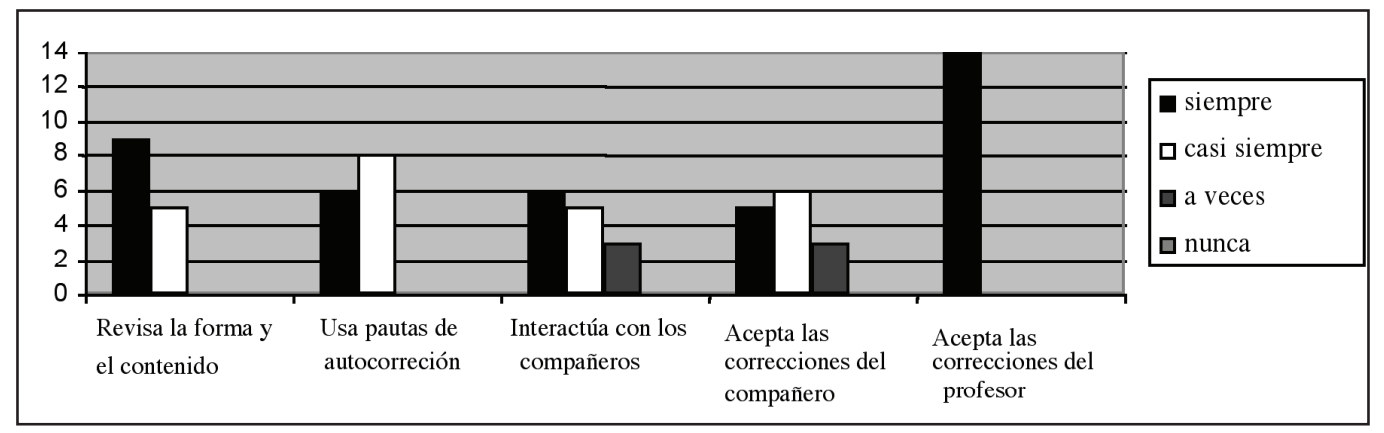

\subsection{Revisa la forma y el contenido}

En muchas ocasiones, a la hora de revisar su propio escrito o el de sus compañeros, el estudiante toma dos posiciones: o solo revisa la forma, es decir, aquellos aspectos relacionados con la gramática, la correspondencia de los tiempos, la ortografía, etc.; o solo revisa el contenido y determina cómo está organizado el texto, si hay coherencia, si está claro el mensaje, etc.

En el enfoque al proceso no se corrige el producto sino el proceso de redacción, es decir, no interesa tanto eliminar las faltas gramaticales, sino que el estudiante mejore sus hábitos de composición. Sin embargo, durante el proceso de revisión, el estudiante comprende los errores de gramática cometidos y los reformula a fin de internalizarlos y no volver a repetirlos. Lo mismo ocurre cuando se pone atención al contenido: el estudiante comprende cómo organizar mejor la información de manera que quede claro el mensaje que se ha propuesto transmitir.

El gráfico No. 3 muestra que la mayoría de los estudiantes, con un porcentaje de $64,28 \%$, se interesa siempre por la revisión de la forma y del contenido, mientras que cinco estudiantes, correspondientes a un $35,71 \%$, lo hacían casi siempre. Las frecuencias a veces y nunca muestran un porcentaje de $0 \%$.

\subsection{Usa pautas de autocorrección}

Cada tarea que el estudiante debía realizar tenía una lista de cotejo al final (véase las unidades del manual adjunto). Estas listas tienen como finalidad ayudar al estudiante a focalizar los puntos que son importantes en la redacción que deben escribir. Además, la revisión se hace más ordenada, con más precisión y, a veces, en menos tiempo. Sin embargo, y a pesar de que se 
le pedía explícitamente que usara esas listas, muchas veces el estudiante no lo hacía y revisaba sus escritos o los de sus compañeros con sus propios recursos.

Este comportamiento se puede observar en el gráfico No. 3, el cual arroja lo siguiente: de los catorce estudiantes, solo seis, con un porcentaje de 42,85, usaban siempre esas listas de cotejo. La mayoría, que corresponde a ocho estudiantes, con un porcentaje de 57,14, solo lo hacía casi siempre, mientras que las frecuencias a veces y nunca no reportaron porcentajes. Estos resultados están intrínsecamente relacionados con los resultados que se explicarán más adelante. Es decir, si los estudiantes no utilizaron las listas de cotejo, ni aceptaban en las correcciones de sus compañeros, probablemente se deba a que ellos preferían las correcciones del profesor.

\subsection{Interactúa con sus compañeros en la revisión}

El estudiante en esta etapa puede revisar su propio escrito o pedirle a su compañero hacerlo. Durante el curso, esta situación dependía de la naturaleza de la tarea que se debía realizar o de la programación de la clase. En cuanto al primer aspecto, algunas tareas se hacían de manera individual, pero se revisaban en conjunto, tales como los ejercicios de complete o de respuesta breve. Otras, por el contrario, debían revisarse en parejas.

Al respecto, los datos del gráfico No. 3 muestran lo siguiente: seis estudiantes, con un porcentaje de 42,85, siempre interactuaban con sus compañeros en la revisión, es decir, se interesaban por lo que su compañero les indicaba, le hacían preguntas, trataban de aclarar sus dudas en conjunto. En la frecuencia casi siempre solamente se reportaron cinco estudiantes, correspondientes a un $35,71 \%$, mientras que la frecuencia a veces fue ocupada por un $21,42 \%$.

Estos datos son interesantes, ya que siempre hubo muy buena relación entre los estudiantes y hubo mucha comunicación entre ellos, especialmente en las actividades orales. Sin embargo, los datos del gráfico parecen indicar que los estudiantes se sentían más cohibidos a la hora de la revisión. Se podría conjeturar que los estudiantes no tenían confianza en sus conocimientos y temían no hacer las correcciones de manera correcta o no querían hacer "públicos" sus errores.

\subsection{Acepta las correcciones hechas por sus compañeros}

Este criterio está en estrecha relación con el anterior. A través de todo el curso, hubo varias clases donde las revisiones se hicieron en grupo; con esto podría pensarse que los estudiantes se sentían cómodos y con confianza para corregir los errores y aceptar las correcciones. Sin embargo, en los datos del gráfico No. 3 se observa que solamente cinco estudiantes, que corresponden a un 35,71\%, aceptaban las correcciones siempre, mientras que un 42,85\% lo hacía casi siempre. Es interesante notar que tanto en el criterio "interactúa con sus compañeros" como en "acepta los errores de sus compañeros", se reportaron tres estudiantes $(21,42 \%)$ en la misma frecuencia de a veces. Se pudo detectar que este caso corresponde específicamente a tres muchachas que tenían vínculos de amistad muy profundos y que, por razones de esa misma amistad, interactuaban más entre ellas y menos con el grupo en general.

\subsection{Acepta las correcciones hechas por el profesor}

En este enfoque, el profesor no cumple un rol tradicional que corrige y ordena, sino que es más bien un asesor o guía que facilita los elementos básicos para que los estudiantes encuentren sus propias estrategias en el proceso de la escritura. El trabajo del profesor en este 
enfoque es muy individualizado: él asesora individualmente al estudiante, le dice qué técnicas puede utilizar, qué debe mejorar en la gramática o en el contenido, cómo puede ordenar la información, etc. Asimismo, el trabajo del estudiante es muy libre; si bien sigue las pautas del profesor, tiene la responsabilidad y el control sobre su propio trabajo.

Los datos del gráfico No. 3 muestran que el 100\% de los estudiantes siempre confió más en el profesor y aceptó sus correcciones, mientras que las demás frecuencias muestran un porcentaje del $0 \%$. Este dato evidencia que, a pesar de todo, existe una jerarquía donde las relaciones del proceso de educación siempre se dan en forma vertical: el profesor tiene el conocimiento, la palabra: él dice y el alumno hace, y en el sistema educativo con esa perspectiva es muy difícil cambiar los papeles. Por esta misma razón, los estudiantes no recurrían a la corrección de los compañeros, prefiriendo las correcciones hechas por el profesor.

\section{Conclusiones}

La práctica de escritura enfocada en el proceso no se caracteriza, en esencia, por la espontaneidad ni por el automatismo, sino por la recursividad, por la reformulación de ideas, por la realización de múltiples borradores y por la revisión constante. En este proyecto se logró comprobar que, mediante las fases del proceso, los estudiantes mejoraron en cuanto al esbozo y la delimitación del tema, el planteamiento apropiado de la introducción, el desarrollo y la conclusión de los textos, la concordancia entre los distintos elementos de la oración, el establecimiento, desde el principio, del propósito y la audiencia, la organización de las ideas y del texto en general.

Tal como lo demuestran los resultados obtenidos en esta investigación, la aplicación de las diversas estrategias determinó un mayor aprovechamiento de los conocimientos aprendidos. Entre estas se encuentran aquellas relacionadas con las técnicas para la generación de ideas, ya que les permitió a los estudiantes hacer planes y esquemas del texto de manera más útil y sencilla. También fue positivo el hecho de que la mayoría de los estudiantes escribió más de un borrador antes de entregar la versión final. Así, en este proyecto se demostró que la corrección es provechosa cuando se realiza durante el proceso de la redacción del texto, es decir, cuando se corrigen los borradores y los esquemas iniciales de manera que el estudiante puede incorporarla en la redacción final.

En cuanto al proceso de corrección, se hizo evidente que la retroalimentación resulta ser primordial para la elaboración de estrategias didácticas. Con esta metodología el estudiante no solo evalúa su propia experiencia, sino que aprende también de los errores de sus compañeros. Aunque los resultados muestran que la mayoría de ellos no utilizaron las listas de cotejo, sino que prefirieron las recomendaciones del profesor, se demostró que el interés por distinguir sus propios errores motivaba el estímulo de la experimentación creativa. De manera que, en vez de tomarlo como un desacierto, el estudiante se dedicaba a la reelaboración de los temas para así aumentar el conocimiento.

En el enfoque al proceso, el papel del profesor se modifica sustancialmente: de inquisidor evaluativo a facilitador activo. La aplicación de este principio en este proyecto permitió que el estudiante compartiera con el instructor la experiencia creativa del aprendizaje y no el temor de la evaluación. Así, la clase se convirtió en un espacio libre y autónomo donde cada uno escribía, a su propio ritmo, los textos determinados en el programa. 
Las actividades previas a la escritura de los diversos textos, tales como las canciones, los videos, las lecturas y las discusiones ayudaron a los estudiantes en el proceso de composición. Estas actividades constituyen una valiosa fuente de estímulo para los estudiantes, ya que no solamente pudieron extraer temas para las redacciones, sino que aumentaron el vocabulario que podía ser usado en la redacción.

Por último, cabe señalar que enfocar el proceso de escritura en el marco del Método Comunicativo permitió integrar la escritura a las otras destrezas, de manera que las actividades escritas estuvieran correlacionadas con el habla, la lectura y la escucha. El desarrollo de las cuatro destrezas incluyó distintas dinámicas grupales que motivaron a los estudiantes a integrarse plenamente en la clase.

\section{Bibliografía}

ACTFL. 1999. Oral Proficiency Interview, Tester training manual. N.Y: American Council on the Teaching of Foreign Languages.

1999. Proficiency Guidelines-Speaking. N.Y: American Council on the Teaching of Foreign Languages.

Brookes, Arthur y P. Grundy. 1998. Beginnig to write: writing activities for elementary and intermediate learners. Cambridge: Cambridge University Press.

Brown, Douglas. 1994. Teaching by Principles: an interactive approach to language pedagogy. New Jersey: Prentice Hall Regents.

2000. Principles of Language Learning and Teaching. New York: Addison Wesley Longman, Inc.

Carduner, Jessie. 2002. "Using Classroom Assessment Techniques to Improve Foreign Language Composition Courses". N. Y: American Council of the Teaching on Foreign Languages. Foreign Language Annals. 35 (5).

Cassany, Daniel. 1989. Describir el escribir. España: Ediciones Paidós Ibérica, S.A.

1999. Construir la escritura. España: Ediciones Paidós Ibérica, S.A.

2000. Reparar la escritura. Didáctica de la corrección de lo escrito. Barcelona: Editorial Graó.

Condemarín, Mabel y Mariana Chadwick. 1999. Taller de Escritura. Santiago de Chile: Editorial Universitaria, S.A.

Ellis, Rod. 1997. SLA Research and Language Teaching. Oxford: Oxford University Press. 
Encina, Alonso. 1986. ¿Cómo ser profesorla y querer seguir siéndolo? Madrid: Edelsa Grupo Didascalia, S.A.

Gerrard, Lisa y Sheri Spaine Long. 1993. Redacción y revisión. United States of America: McGraw-Hill, Inc.

Harmer, Jeremy. 1987. Teaching and learning grammar. New York: Longman Publishing.

Jurado Spuch, Adelaida. 2005. "Writing activities should be as much concerned with process as with product". En: Actas II, IX Simposio Internacional de Comunicación Social. Centro de Lingüística Aplicada. Santiago de Cuba.

Larsen-Freeman. 1986. Techniques and principles in language teaching. Oxfor: Oxford University Press.

Llobera, M. 1995. Competencia comunicativa. Documentos básicos en la enseñanza de lenguas extranjeras. Madrid:Edelsa Grupo Didascalia, S.A.

Madrigal Abarca, Marta. 2005. "La enseñanza de la escritura como proceso a estudiantes de español como segunda lengua de nivel avanzado". Informe final de práctica dirigida. Universidad de Costa Rica.

Manchón, R., Roca, J. y L. Murphy. 2000. "La influencia de la variable <grado de dominio de la L2> en los procesos de composición en lengua extranjera: hallazgos recientes de investigación”. En: Muñoz, Carmen (comp.).

Nance K, y I. Rivera. 1996. Técnicas de composición. Massachussets: D.C. Heath and Company.

Navarro Thames, Carlos. 1998. “Técnicas para la corrección de errores del habla en cursos de idiomas". Kañina. 22 (2): 103- 112.

Nunan, David. 1999. Second Language Teaching and Learning. Boston: Heinkle \& Heinkle Publishers.

Omaggio Hadley, Alice. 1993. Teaching Language in Context. Boston: Heinle \& Heinle Publishers.

Richards, Jack C. y T. Rodgers. 1998. Enfoques y métodos en la enseñanza de idiomas. Cambridge: Cambridge University Press.

Richards, Jack C. 1990. The Language Matrix. Cambridge: Cambridge University Press.

2001. Curriculum development in language teaching. Cambridge: Cambridge University Press. 
Sánchez Pérez, Aquilino. 1997. Los métodos en la enseñanza de idiomas. España: Sociedad General Española de Librería, S.A.

Solís, Mayra. 1998. "Developing student's writing skills in english through systematic grammar exercises and teachers's feedback". Káñina. 22 (2): 125- 132.

Tardy, William. 1994. Composiciones ilustradas. Illinois: Nacional Textbook Company. Espéculo. Núm. 15. Madrid: Universidad Complutense de Madrid.

Ur, Penny. 1995. A course in Language Teaching. Practice and theory. Cambridge: Cambridge University. 